\title{
Minimum Diameter Spanning Tree
}

\author{
V. T. Chandrasekaran1, N. Rajasri ${ }^{2}$ \\ ${ }^{1}$ Department of Mathematics, Jawahar Science College, Neyveli, India \\ ${ }^{2}$ Department of Mathematics, Vallalar Arts and Science College, Vadalur, India \\ Email: vtcvtc14@gmail.com, rajasri150583@gmail.com
}

How to cite this paper: Chandrasekaran, V.T. and Rajasri, N. (2018) Minimum Diameter Spanning Tree. American Journal of Computational Mathematics, 8, 203-208. https://doi.org/10.4236/ajcm.2018.83016

Received: August 16, 2018

Accepted: September 4, 2018

Published: September 7, 2018

Copyright $\odot 2018$ by authors and Scientific Research Publishing Inc. This work is licensed under the Creative Commons Attribution International License (CC BY 4.0).

http://creativecommons.org/licenses/by/4.0/

\begin{abstract}
In this paper, we discuss the simple connected graphs which have a minimum diameter spanning tree such that both have same domination number.
\end{abstract}

\section{Keywords}

Diameter, Spanning Tree, Essential Dominating Vertex, Pan Graph, Ladar Graph

\section{Preliminaries}

E.J. Cockayne and S.T. Hedetniemi [1] introduced the concept dominating set. Frank Harary, Robert Z.Norman and Dorwin Cartwright [2] explained an interesting application in voting situations using the concept of domination. C.L. Liu [3] also discussed the application of dominance to communication network, where a dominating set represents a set of cities which acting as transmitting stations, can transmit messages to every city in the network.

A subset $S$ of vertices from $V$ is called a dominating set for $G$ if every vertex of $G$ is either a member of $S$ or adjacent to a member of $S$. A dominating set of $G$ is called a minimum dominating set if $G$ has no dominating set of smaller cardinality. The cardinality of minimum dominating set of $G$ is called the dominating number for $G$ and it is denoted by $\gamma(G)$ [4].

The eccentricity of a graph vertex $v$ in a connected graph $G$ is the maximum graph distance between $v$ and any other vertex $u$ of $G$. The eccentricity $e(v)$ of $v$ is defined by $e(v)=\max \{d(u, v): u \in V(G)\}$. The radius of a graph $r(G)$ is defined by $r(G)=\min \{e(v): v \in V(G)\}$. The diameter of a graph $d(G)$ is defined by $d(G)=\max \{e(v): v \in V(G)\} . \quad V$ is called a centre point if $e(v)=r(G)$ and the set of all central point is called the centre of $G$. A spanning tree is a subset of a graph $G$, which has all the vertices covered with minimum possible number of edges, hence a spanning tree doesn't have a cycle and it can't be disconnected. A spanning tree of a graph $G(V, E)$ with minimum diameter 
is called the minimum diameter spanning tree.

M. Yamuna and K. Karthika [5] provided a constructive procedure to generate a spanning tree for any graph from its dominating set, $\gamma$-set.

In this paper, we discuss few simple connected graphs for which the domination numbers of the graph and that of its minimum diameter spanning tree are the same.

\section{Some Definitions}

In this section, we present few definitions and examples which are required for this article.

Definition 1. The spanning tree $T$ of the simple connected graph $G$ is said to be a minimum diameter spanning tree if there is no other spanning tree $T^{\prime}$ of $G$ such that $d\left(T^{\prime}\right)<d(T)$.

Definition 2. A vertex $u$ of a simple connected graph is an essential dominating vertex, if every minimum dominating set contains the vertex $u$.

Example 1. In the graph given in Figure 1, the vertex $u$ is an essential dominating vertex.

Definition 3. [6] The pan graph is the graph obtained by joining a cycle graph $C_{n}$ to a singleton graph $K_{1}$ with a bridge and we denote it by $\mathcal{P}_{n}$.

Example 2. The graphs given in Figure 2 are pan graphs.

Definition 4. [6] The ladder graph $L_{n}$ is a planar undirected graph with $2 n$ vertices and $n+2(n-1)$ edges.

Example 3. The graph given in Figure 3 is a ladar graph $L_{7}$.

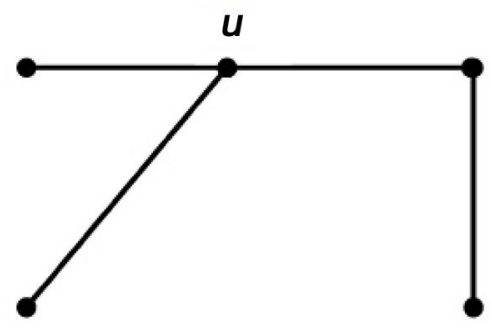

Figure 1. A Simple connected graph with essential dominating vertex $u$.
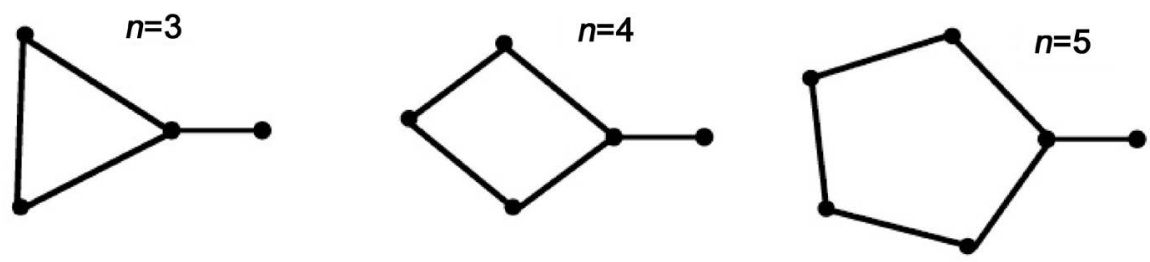

Figure 2. Pan Graphs.

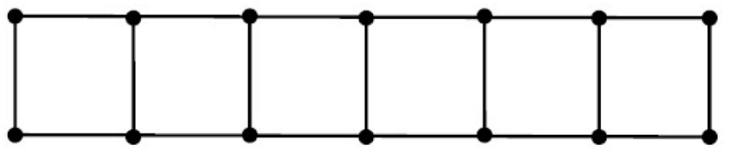

Figure 3. Ladar Graph $L_{7}$. 


\section{Some Results}

In this section we discuss the graphs which have minimum diameter spanning trees such that both have same domination number. It is clear to observe that cycle, regular graph, complete bipartite graph have minimum diameter spanning tree, possessing domination number same that of the graph.

Proposition 1. Every pan graph has at least one essential dominating vertex. In fact, the vertex, which is adjacent to the pendent vertex is an essential dominating vertex.

Proof: By definition 3 of a pan graph, it is a cycle in which one of the nodes in the cycle is adjacent to a node which is not in the cycle. Let $u$ be the such vertex in the cycle, which is adjacent to the pendent vertex $v$. Since $u$ is in the cycle, $u$ is also adjacent to two other vertices, say $x$ and $y$, in the cycle. Clearly, the vertex $u$ dominates the three vertices $v, x$ and $y$. Suppose $u$ is not an essential dominating set, then there must exist another vertex in the cycle, which dominates the four vertices $u, V, x$ and $y$. But obviously, no such vertex exists. Hence, $u$ is an essential dominating vertex.

Let us recall the following results $\gamma\left(\mathcal{P}_{n}\right)=\left\lfloor\frac{n+2}{3}\right\rfloor$ and $\gamma\left(P_{n}\right)=\left\lfloor\frac{n+2}{3}\right\rfloor$

Proposition 2. A pan graph $\mathcal{P}_{n}$ has a minimum diameter spanning tree $T$ for which $\gamma\left(\mathcal{P}_{n}\right)=\gamma(T)$ if $n \neq 0$ or $5(\bmod 6)$

Proof: Consider $\mathcal{P}_{n}$ where $n>3$. To obtain a spanning tree of $\mathcal{P}_{n}$, we must remove an edge from the cycle. To obtain a minimum diameter spanning tree $T$, we must remove an edge which is far away from the pendant vertex. In other words, the path $P_{n-3}$, obtained after the removal of the vertex $u$, must be separated in to two paths of length equal or one path has length 1 more than the other. Now, let us discuss the following cases.

Case $1: n \equiv 0(\bmod 6)$

Let $n=6 m$ for some positive integer. Then $n-3=6 m-3$. Therefore the path $P_{n-3}=P_{6 m-3}$ can be separated into two paths $P_{3 m-2}$ and $P_{3 m-1}$. Since $\gamma\left(P_{3 m-2}\right)=\left\lfloor\frac{3 m}{3}\right\rfloor=m$ and $\gamma\left(P_{3 m-1}\right)=\left\lfloor\frac{3 m+1}{3}\right\rfloor=m$, we have $\gamma(T)=2 m+1$. But $\gamma\left(\mathcal{P}_{n}\right)=\left\lfloor\frac{6 m+2}{3}\right\rfloor=2 m$.

Case 2: $n \equiv 1(\bmod 6)$

Let $n=6 m+1$ for some positive integer. Then $n-3=6 m-2$. Therefore the path $P_{n-3}=P_{6 m-2}$ can be separated into two equal paths $P_{3 m-1}$. But $\gamma\left(P_{3 m-1}\right)=\left\lfloor\frac{3 m+1}{3}\right\rfloor=m$ and hence we have $\gamma(T)=2 m+1$. Also $\gamma\left(\mathcal{P}_{n}\right)=\left\lfloor\frac{6 m+3}{3}\right\rfloor=2 m+1$

Case 3: $n \equiv 2(\bmod 6)$

Let $n=6 m+2$ for some positive integer. Then $n-3=6 m-1$. Therefore the path $P_{n-3}=P_{6 m-1}$ can be separated into two paths $P_{3 m-1}$ and $P_{3 m}$. But 
$\gamma\left(P_{3 m-1}\right)=\left\lfloor\frac{3 m+1}{3}\right\rfloor=m$ and $\gamma\left(P_{3 m}\right)=\left\lfloor\frac{3 m+2}{3}\right\rfloor=m$, and hence we have $\gamma(T)=2 m+1$. Also $\gamma\left(\mathcal{P}_{n}\right)=\left\lfloor\frac{6 m+4}{3}\right\rfloor=2 m+1$.

Case 4: $n \equiv 3(\bmod 6)$

Let $n=6 m+3$ for some positive integer. Then $n-3=6 m$. Therefore the path $P_{n-3}=P_{6 m}$ can be separated into two equal paths $P_{3 m}$. But

$\gamma\left(P_{3 m}\right)=\left\lfloor\frac{3 m+2}{3}\right\rfloor=m$, hence $\gamma(T)=2 m+1$. Also $\gamma\left(\mathcal{P}_{n}\right)=\left\lfloor\frac{6 m+5}{3}\right\rfloor=2 m+1$.

Case 5: $n \equiv 4(\bmod 6)$

Let $n=6 m+4$ for some positive integer. Then $n-3=6 m+1$. Therefore the path $P_{n-3}=P_{6 m+1}$ can be separated into two paths $P_{3 m}$ and $P_{3 m+1}$. But $\gamma\left(P_{3 m}\right)=\left\lfloor\frac{3 m+2}{3}\right\rfloor=m$ and $\gamma\left(P_{3 m+1}\right)=\left\lfloor\frac{3 m+3}{3}\right\rfloor=m+1$, hence $\gamma(T)=2 m+2$. Also $\gamma\left(\mathcal{P}_{n}\right)=\left\lfloor\frac{6 m+6}{3}\right\rfloor=2 m+2$.

Case 6: $n \equiv 5(\bmod 6)$

Let $n=6 m+5$ for some positive integer. Then $n-3=6 m+2$. Therefore the path $P_{n-3}=P_{6 m+2}$ can be separated into two equal paths $P_{3 m+1}$. But

$\gamma\left(P_{3 m+1}\right)=\left\lfloor\frac{3 m+3}{3}\right\rfloor=m+1$, hence we have $\gamma(T)=2 m+3$. But

$\gamma\left(\mathcal{P}_{n}\right)=\left\lfloor\frac{6 m+7}{3}\right\rfloor=2 m+2$.

Hence the proof of the proposition follows.

Proposition 3. The ladder graphs $L_{n}$ have minimum diameter spanning tree $T$ such that $\gamma\left(L_{n}\right)=\gamma(T)$, only when $n \leq 3$ or $n$ is an even integer.

Proof: In a ladar graph $L_{n}$, the $2 n$ vertices are divided in to two sets of each $n$ vertices and each vertex of one set is adjacent to exactly one vertex of the other set and vice-versa. We may call these edges as step edges. Further, each of the $n$ vertices are connected by a path on $n-1$ edges. We may note that the diameter of a minimum diameter spanning tree of $L_{n}$ is $n$, when $n$ is odd and $n+1$, when $n$ is even. In fact when $n$ is odd, $L_{n}$ has a unique minimum diameter spanning tree, which is obtained by deleting all the step edges, except the middle one. When $n$ is even, there may be several minimum diameter spanning trees. The Figure 4 \& Figure 5 respectively illustrate the minimum diameter spanning trees of $L_{5}$ and $L_{6}$.

Further $\gamma\left(L_{n}\right)=1+\left\lfloor\frac{n}{2}\right\rfloor$.

Now let us consider the two cases on $n$

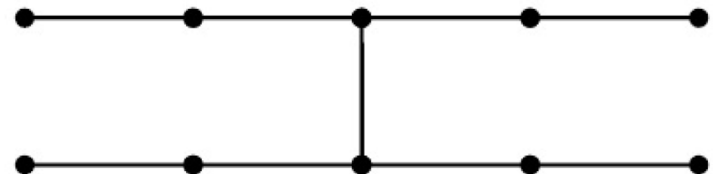

Figure 4. Unique minimum diameter spanning tree of $L_{5}$. 

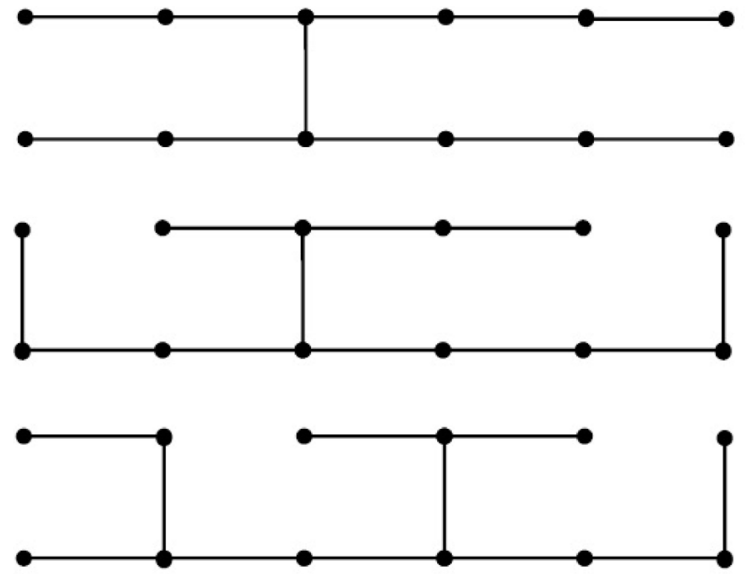

Figure 5. Minimum diameter spanning trees of $L_{6}$.

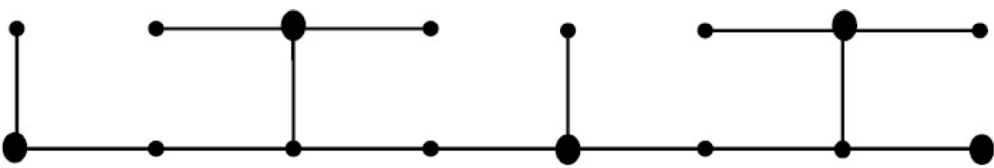

Figure 6. A Minimum diameter spanning trees of $L_{8}$ with Equal dominating numbers.

Case: $1 n$ is odd.

When $n$ is odd, the domination number of the unique minimum diameter spanning tree of $L_{n}$ is given by $2 \times\left\lfloor\frac{n}{2}\right\rfloor$. But this is equal to $1+\left\lfloor\frac{n}{2}\right\rfloor$ only when $n=3$.

Case: $2 n$ is even.

When $n$ is even, the domination number of the minimum diameter spanning tree of $L_{n}$ is given by $2+\left\lfloor\frac{2 n-1}{4}\right\rfloor$, because in a ladar graph, a node can dominate maximum four nodes, including itself. Since $2 n$ is divisible by 4 , the dominated nodes can be grouped with 4 nodes. The final 4 nodes can be found as 1 in one end and 3 in the other end. The 1 node is dominated by itself and in the 3 nodes, one node dominates the other two. Also this is equal to $1+\left\lfloor\frac{n}{2}\right\rfloor$. Hence the proof follows.

An example is given in Figure 6.

\section{Conclusion}

In this article, we have discussed few graphs for which the domination number is the same that of its minimum diameter spanning tree. Further research can be done in exploring various graphs with the same property. The condition for which a graph does not posses such spanning tree may also be explored.

\section{Conflicts of Interest}

The authors declare no conflicts of interest regarding the publication of this paper. 


\section{References}

[1] Cockayne, E.J. and Hedetniemi, S.T. (1977) Towards a Theory of Domination in Graphs. Networks, 7, 247-261. https://doi.org/10.1002/net.3230070305

[2] Harary, F., Norman, R.Z. and Cartwright, D. (1967) An Introduction to the Theory of Directed Graphs. John Wiley and Sons, New York.

[3] Liu, C.L. (1968) Introduction to Combinatorial Mathematics. McGraw-Hill, New York.

[4] Haray, F. (1969) Graph Theory. Addsion Wesley, Reading, MA. https://doi.org/10.21236/AD0705364

[5] Yamuna, M. and Karthika, K. (2013) Minimal Spanning Tree from a Minimum Dominating Set. WSEAS Transactions on Mathematics, 12, 1055-1064.

[6] Bray, N. and Weisstein, E.W. (2018) Domination Number. MathWorld-A Wolfram Web Resource, Champaign. 